\title{
On periodic-type boundary value problems for functional differential equations with positively homogeneous operator
}

\author{
Robert Hakl
}




\title{
ON PERIODIC-TYPE BOUNDARY VALUE PROBLEMS FOR FUNCTIONAL DIFFERENTIAL EQUATIONS WITH A POSITIVELY HOMOGENEOUS OPERATOR
}

\author{
ROBERT HAKL
}

[Received: September 10, 2003]

\begin{abstract}
Consider the problem
$u^{\prime}(t)=H(u)(t)+Q(u)(t), \quad u(a)-\lambda u(b)=h(u)$,

where $H, Q: C([a, b] ; \mathbb{R}) \rightarrow L([a, b] ; \mathbb{R})$ are continuous operators satisfying the Carathèodory condition, the operator $H$ is positively homogeneous, $\lambda \in \mathbb{R}_{+}$, and $h: C([a, b] ; \mathbb{R}) \rightarrow \mathbb{R}$ is a continuous functional. In this paper, efficient sufficient conditions guaranteeing the solvability and unique solvability of the problem considered are established.
\end{abstract}

Mathematics Subject Classification: 34K06, 34K10

Keywords: Functional differential equation, boundary value problem, periodic solution

\section{INTRODUCTION}

$I^{N}$ THIS PAPER, we establish efficient sufficient conditions guaranteeing the existence and uniqueness of a solution of a periodic-type boundary value problem for a scalar functional differential equation involving a positively homogeneous operator.

The following notation is used throughout the paper.

$\mathbb{R}$ is the set of all real numbers, $\mathbb{R}_{+}=\left[0,+\infty\left[,[x]_{+}=\frac{1}{2}(|x|+x),[x]_{-}=\frac{1}{2}(|x|-x)\right.\right.$. $C([a, b] ; \mathbb{R})$ is the Banach space of continuous functions $u:[a, b] \rightarrow R$ with the norm $\|u\|_{C}=\max \{|u(t)|: t \in[a, b]\}$.

$\tilde{C}([a, b] ; \mathbb{R})$ is the set of absolutely continuous functions $u:[a, b] \rightarrow \mathbb{R}$.

$L([a, b] ; \mathbb{R})$ is the Banach space constituted by the Lebesgue integrable functions $p:[a, b] \rightarrow \mathbb{R}$ with the norm $\|p\|_{L}=\int_{a}^{b}|p(s)| d s$.

$L\left([a, b] ; \mathbb{R}_{+}\right)=\{p \in L([a, b] ; \mathbb{R}): p(t) \geq 0$ for $t \in[a, b]\}$.

$\mathcal{M}_{a b}$ is the set of measurable functions $\tau:[a, b] \rightarrow[a, b]$.

$\mathcal{K}_{a b}$ is the set of continuous operators $F: C([a, b] ; \mathbb{R}) \rightarrow L([a, b] ; \mathbb{R})$ satisfying the Carathèodory condition, i. e., for every $r>0$ there exists $q_{r} \in L\left([a, b] ; \mathbb{R}_{+}\right)$such 
that

$$
|F(v)(t)| \leq q_{r}(t) \quad \text { for } t \in[a, b], \quad v \in C([a, b] ; \mathbb{R}), \quad\|v\|_{C} \leq r .
$$

$\mathcal{H}_{a b}$ is the set of operators $H \in \mathcal{K}_{a b}$ which are positively homogeneous, i. e., such that, for an arbitrary number $\alpha>0$, we have

$$
H(\alpha v)(t)=\alpha H(v)(t) \quad \text { for } t \in[a, b], \quad v \in C([a, b] ; \mathbb{R}) .
$$

$\mathcal{P}_{a b}$ is the set of operators $H \in \mathcal{H}_{a b}$ which are nondecreasing, i. e., for all $H \in \mathcal{P}_{a b}$ and $u, v \in C([a, b] ; \mathbb{R})$ such that $u(t) \leq v(t)$ for $t \in[a, b]$, we have

$$
H(u)(t) \leq H(v)(t) \quad \text { for } t \in[a, b] .
$$

$K\left([a, b] \times \mathbb{R}_{+} ; \mathbb{R}_{+}\right)$is the set of functions $\eta:[a, b] \times \mathbb{R}_{+} \rightarrow \mathbb{R}_{+}$satisfying the Carathèodory conditions, i. e., $\eta(\cdot, x):[a, b] \rightarrow \mathbb{R}_{+}$is a measurable function for all $x \in \mathbb{R}_{+}, \eta(t, \cdot): \mathbb{R}_{+} \rightarrow \mathbb{R}_{+}$is a continuous function for almost all $t \in[a, b]$, and for every $r>0$ there exists $q_{r} \in L\left([a, b] ; \mathbb{R}_{+}\right)$such that

$$
|\eta(t, x)| \leq q_{r}(t) \quad \text { for } t \in[a, b], \quad 0 \leq x \leq r .
$$

By a solution of the equation

$$
u^{\prime}(t)=H(u)(t)+Q(u)(t)
$$

where $H \in \mathcal{H}_{a b}$ and $Q \in \mathcal{K}_{a b}$, we understand a function $u \in \tilde{C}([a, b] ; \mathbb{R})$ satisfying equality (1.1) almost everywhere in $[a, b]$.

Consider the problem on the existence and uniqueness of a solution of (1.1) satisfying the condition

$$
u(a)-\lambda u(b)=h(u)
$$

where $h: C([a, b] ; \mathbb{R}) \rightarrow \mathbb{R}$ is a continuous operator such that for every $r>0$ there exists $M_{r} \in \mathbb{R}_{+}$such that

$$
|h(v)| \leq M_{r} \quad \text { for } v \in C([a, b] ; \mathbb{R}), \quad\|v\|_{C} \leq r .
$$

The differential equations with "maxima" are one of the special cases of equation (1.1). Various types of boundary value problems for this type of equations were studied, e. g., in $[3,4,33,35,36,38,41]$.

In the case where $H$ in (1.1) is a linear operator, the results presented here coincide with those obtained earlier in $[6,17]$ (see also [22]). Another type of conditions guaranteeing the solvability of (1.1), (1.2) with a linear operator $H$ can be found, e. g., in $[5,8,10-15,18-21,28,34]$. Conditions for the solvability and unique solvability for other types of boundary value problems for equation (1.1) with a linear operator $H$ are established, e. g., in $[9,16,22,24,27,39,42]$.

There are many interesting results concerning the solvability of general boundary value problems for functional differential equations (see, e. g., [1,2, 7,9,22-26,29-32, $37,40]$ and references therein). In spite of this, the general theory of boundary value problems for functional differential equations is not still complete. Here, we try to fill this gap in a certain way. More precisely, in Section 2, we establish unimprovable, 
in a certain sense, efficient conditions sufficient for the solvability of problem (1.1), (1.2). In Section 3, we prove some auxiliary propositions. Section 4 is devoted to the proofs of the main results.

The results are concretized for the boundary value problem of the form

$$
\begin{gathered}
u^{\prime}(t)=p(t) \max \left\{u(s): \tau_{1}(t) \leq s \leq \tau_{2}(t)\right\}+q(t), \\
u(a)-\lambda u(b)=c,
\end{gathered}
$$

where $p, q \in L([a, b] ; \mathbb{R}), \tau_{1}, \tau_{2} \in \mathcal{M}_{a b}, \tau_{1}(t) \leq \tau_{2}(t)$ for $t \in[a, b]$, and $c \in \mathbb{R}$.

Definition 1.1. We will say that an operator $H \in \mathcal{K}_{a b}$ is subadditive if for any $u, v \in$ $C([a, b] ; \mathbb{R})$ we have

$$
H(u+v)(t) \leq H(u)(t)+H(v)(t) \quad \text { for } t \in[a, b] .
$$

Definition 1.2. We will say that an operator $H \in \mathcal{K}_{a b}$ is superadditive if for any $u, v \in C([a, b] ; \mathbb{R})$ we have

$$
H(u+v)(t) \geq H(u)(t)+H(v)(t) \quad \text { for } t \in[a, b] .
$$

\section{MaIn Results}

Throughout the paper, $\eta \in K\left([a, b] \times \mathbb{R}_{+} ; \mathbb{R}_{+}\right)$and $\eta_{0}: \mathbb{R}_{+} \rightarrow \mathbb{R}_{+}$are such that

$$
\lim _{x \rightarrow+\infty}\left(\frac{\eta_{0}(x)}{x}+\frac{1}{x} \int_{a}^{b} \eta(s, x) d s\right)=0 .
$$

Theorem 2.1. Let $\lambda \in] 0,1]$ and let $H$ admit the representation $H=H_{0}-H_{1}$, where $H_{0}, H_{1} \in \mathcal{P}_{a b}$. Let, furthermore,

$$
\begin{gathered}
|Q(v)(t)| \leq \eta\left(t,\|v\|_{C}\right) \quad \text { for } t \in[a, b], \quad v \in C([a, b] ; \mathbb{R}), \\
|h(v)| \leq \eta_{0}\left(\|v\|_{C}\right) \quad \text { for } v \in C([a, b] ; \mathbb{R}) .
\end{gathered}
$$

If, moreover,

$$
\begin{gathered}
\int_{a}^{b} H_{0}(1)(s) d s<1, \quad \int_{a}^{b}\left|H_{0}(-1)(s)\right| d s<1, \\
\frac{\int_{a}^{b} H_{0}(1)(s) d s}{1-\int_{a}^{b} H_{0}(1)(s) d s}-\frac{1-\lambda}{\lambda}<\int_{a}^{b} H_{1}(1)(s) d s, \\
\frac{\int_{a}^{b}\left|H_{0}(-1)(s)\right| d s}{1-\int_{a}^{b}\left|H_{0}(-1)(s)\right| d s}-\frac{1-\lambda}{\lambda}<\int_{a}^{b}\left|H_{1}(-1)(s)\right| d s,
\end{gathered}
$$




$$
\begin{aligned}
{\left[\int_{s}^{t} H_{1}(1)(\xi) d \xi\right.} & -1]_{+}\left[\int_{I}\left|H_{1}(-1)(\xi)\right| d \xi-\lambda\right]_{+}< \\
< & \left.<1-\int_{s}^{t}\left|H_{0}(-1)(\xi)\right| d \xi\right)\left(1-\int_{I} H_{0}(1)(\xi) d \xi\right) \\
& \text { for } s, t \in[a, b], \quad s \leq t
\end{aligned}
$$

and

$$
\begin{aligned}
& {\left[\int_{s}^{t}\left|H_{1}(-1)(\xi)\right| d \xi-1\right]_{+}\left[\int_{I} H_{1}(1)(\xi) d \xi-\lambda\right]_{+}<} \\
& <\left(1-\int_{s}^{t} H_{0}(1)(\xi) d \xi\right)\left(1-\int_{I}\left|H_{0}(-1)(\xi)\right| d \xi\right) \\
& \quad \text { for } s, t \in[a, b], \quad s \leq t
\end{aligned}
$$

where $I=[a, b] \backslash] s, t[$, then problem (1.1), (1.2) has at least one solution.

The following assertion can be regarded as a supplement to the previous theorem in the case when $\lambda=0$.

Theorem 2.1'. Let $H$ admit the representation $H=H_{0}-H_{1}$, where $H_{0}, H_{1} \in \mathcal{P}_{a b}$. Let, furthermore, (2.1) and (2.2) be fulfilled. If, moreover, the inequalities (2.3),

$$
\begin{aligned}
& \int_{I}\left|H_{1}(-1)(\xi)\right| d \xi {\left[\int_{s}^{t} H_{1}(1)(\xi) d \xi-1\right]_{+}<} \\
&<\left(1-\int_{s}^{t}\left|H_{0}(-1)(\xi)\right| d \xi\right)\left(1-\int_{I} H_{0}(1)(\xi) d \xi\right) \\
& \text { for } s, t \in[a, b], \quad s \leq t,
\end{aligned}
$$

and

$$
\begin{aligned}
\int_{I} H_{1}(1)(\xi) d \xi\left[\int_{s}^{t}\left|H_{1}(-1)(\xi)\right| d \xi-1\right]_{+}< & \\
& <\left(1-\int_{s}^{t} H_{0}(1)(\xi) d \xi\right)\left(1-\int_{I}\left|H_{0}(-1)(\xi)\right| d \xi\right) \\
& \text { for } s, t \in[a, b], \quad s \leq t
\end{aligned}
$$

are fulfilled, where $I=[a, b] \backslash] s, t[$, then problem (1.1), (1.2) with $\lambda=0$ has at least one solution. 
Theorem 2.2. Let $\lambda \in] 0,1]$ and let $H$ admit the representation $H=H_{0}-H_{1}$, where $H_{0}, H_{1} \in \mathcal{P}_{a b}$. Let, furthermore, (2.1) and (2.2) be fulfilled. If, moreover,

$$
\begin{gathered}
\int_{a}^{b} H_{1}(1)(s) d s<\lambda, \quad \int_{a}^{b}\left|H_{1}(-1)(s)\right| d s<\lambda, \\
\frac{1}{\lambda-\int_{a}^{b} H_{1}(1)(s) d s}-1<\int_{a}^{b} H_{0}(1)(s) d s \\
\frac{1}{\lambda-\int_{a}^{b}\left|H_{1}(-1)(s)\right| d s}-1<\int_{a}^{b}\left|H_{0}(-1)(s)\right| d s \\
{\left[\int_{I} H_{0}(1)(\xi) d \xi-1\right]_{+}\left[\int_{s}^{t}\left|H_{0}(-1)(\xi)\right| d \xi-1\right]_{+}<} \\
<\left(\lambda-\int_{I}\left|H_{1}(-1)(\xi)\right| d \xi\right)\left(1-\int_{s}^{t} H_{1}(1)(\xi) d \xi\right)
\end{gathered}
$$

and

$$
\begin{aligned}
{\left[\int_{I}\left|H_{0}(-1)(\xi)\right| d \xi-1\right]_{+}\left[\int_{s}^{t} H_{0}(1)(\xi) d \xi-1\right]_{+}<} & \\
<\left(\lambda-\int_{I} H_{1}(1)(\xi) d \xi\right)\left(1-\int_{s}^{t}\left|H_{1}(-1)(\xi)\right| d \xi\right) & \text { for } s, t \in[a, b], \quad s \leq t,
\end{aligned}
$$

where $I=[a, b] \backslash] s, t[$, then problem (1.1), (1.2) has at least one solution.

Theorem 2.3. Let $\lambda \in] 0,1]$ and let $H$ admit the representation $H=H_{0}-H_{1}$, where $H_{0}, H_{1} \in \mathcal{P}_{a b}$. Let, furthermore, each of the operators $H_{0}$ and $H_{1}$ be either $a$ subadditive or a superadditive operator, and let there exist $q \in L([a, b] ; \mathbb{R})$ and $c \in \mathbb{R}$ such that

$$
\begin{gathered}
Q(v)(t)=q(t) \quad \text { for } t \in[a, b], \quad v \in C([a, b] ; \mathbb{R}), \\
h(v)=c \quad \text { for } v \in C([a, b] ; \mathbb{R}) .
\end{gathered}
$$

If, moreover,

$$
\begin{gathered}
\int_{a}^{b} \bar{H}_{0}(1)(s) d s<1 \\
\frac{\int_{a}^{b} \bar{H}_{0}(1)(s) d s}{1-\int_{a}^{b} \bar{H}_{0}(1)(s) d s}-\frac{1-\lambda}{\lambda}<\int_{a}^{b}\left|\bar{H}_{1}(-1)(s)\right| d s
\end{gathered}
$$




$$
\begin{aligned}
{\left[\int_{s}^{t} \bar{H}_{1}(1)(\xi) d \xi-1\right]_{+}\left[\int_{I} \bar{H}_{1}(1)(\xi) d \xi-\lambda\right]_{+}<} & \\
<\left(1-\int_{s}^{t} \bar{H}_{0}(1)(\xi) d \xi\right)\left(1-\int_{I} \bar{H}_{0}(1)(\xi) d \xi\right) & \text { for } s, t \in[a, b], \quad s \leq t
\end{aligned}
$$

where $I=[a, b] \backslash] s, t[$ and

$$
\bar{H}_{i}(v)(t) \stackrel{\text { def }}{=} \begin{cases}H_{i}(v)(t) & \text { if } H_{i} \text { is subadditive } \\ -H_{i}(-v)(t) & \text { if } H_{i} \text { is superadditive }\end{cases}
$$

for $t \in[a, b]$ and $i=0,1$, then problem (1.1), (1.2) has a unique solution.

The following assertion can be regarded as a supplement of the previous theorem in the case when $\lambda=0$.

Theorem 2.3'. Let $H$ admit the representation $H=H_{0}-H_{1}$, where $H_{0}, H_{1} \in \mathcal{P}_{a b}$. Let, furthermore, each of the operators $H_{0}$ and $H_{1}$ be either a subadditive or a superadditive operator, and let there exist $q \in L([a, b] ; \mathbb{R})$ and $c \in \mathbb{R}$ such that (2.15) and (2.16) are fulfilled. If, moreover, inequalities (2.17) and

$$
\begin{aligned}
\int_{I} \bar{H}_{1}(1)(\xi) d \xi\left[\int_{s}^{t} \bar{H}_{1}(1)(\xi) d \xi-1\right]_{+}< & \\
<\left(1-\int_{s}^{t} \bar{H}_{0}(1)(\xi) d \xi\right)\left(1-\int_{I} \bar{H}_{0}(1)(\xi) d \xi\right) & \text { for } s, t \in[a, b], \quad s \leq t
\end{aligned}
$$

hold, where $I=[a, b] \backslash] s, t\left[\right.$, and $\bar{H}_{i}(i=0,1)$ are given by (2.20), then problem (1.1), (1.2) with $\lambda=0$ has a unique solution.

Theorem 2.4. Let $\lambda \in] 0,1]$ and let $H$ admit the representation $H=H_{0}-H_{1}$, where $H_{0}, H_{1} \in \mathcal{P}_{a b}$. Let, furthermore, each of the operators $H_{0}$ and $H_{1}$ be either a subadditive or a superadditive operator, and let there exist $q \in L([a, b] ; \mathbb{R})$ and $c \in \mathbb{R}$ such that (2.15) and (2.16) are fulfilled. If, moreover,

$$
\begin{gathered}
\int_{a}^{b} \bar{H}_{1}(1)(s) d s<\lambda \\
\frac{1}{\lambda-\int_{a}^{b} \bar{H}_{1}(1)(s) d s}-1<\int_{a}^{b}\left|\bar{H}_{0}(-1)(s)\right| d s
\end{gathered}
$$




$$
\begin{aligned}
{\left[\int_{I} \bar{H}_{0}(1)(\xi) d \xi-1\right]_{+}\left[\int_{s}^{t} \bar{H}_{0}(1)(\xi) d \xi-1\right]_{+}<} \\
<\left(\lambda-\int_{I} \bar{H}_{1}(1)(\xi) d \xi\right)\left(1-\int_{s}^{t} \bar{H}_{1}(1)(\xi) d \xi\right) \\
\quad \text { for } s, t \in[a, b], \quad s \leq t,
\end{aligned}
$$

where $I=[a, b] \backslash] s, t\left[\right.$, and $\bar{H}_{i}(i=0,1)$ are given by (2.20), then problem (1.1), (1.2) has a unique solution.

Corollary 2.1. Let $\lambda \in] 0,1]$ and let the inequalities

$$
\begin{gathered}
\int_{a}^{b}[p(s)]_{+} d s<1, \\
\frac{\int_{a}^{b}[p(s)]_{+} d s}{1-\int_{a}^{b}[p(s)]_{+} d s}-\frac{1-\lambda}{\lambda}<\int_{a}^{b}[p(s)]_{-} d s<1+\lambda+2 \sqrt{1-\int_{a}^{b}[p(s)]_{+} d s}
\end{gathered}
$$

be fulfilled. Then problem (1.3), (1.4) has a unique solution.

Corollary 2.1'. Let inequalities (2.25) and

$$
\int_{a}^{b}[p(s)]_{-} d s<1+2 \sqrt{1-\int_{a}^{b}[p(s)]_{+} d s}
$$

be fulfilled. Then problem (1.3), (1.4) with $\lambda=0$ has a unique solution.

Corollary 2.2. Let $\lambda \in] 0,1]$ and let the inequalities

$$
\begin{gathered}
\int_{a}^{b}[p(s)]_{-} d s<\lambda, \\
\frac{1}{\lambda-\int_{a}^{b}[p(s)]_{-} d s}-1<\int_{a}^{b}[p(s)]_{+} d s<2+2 \sqrt{\lambda-\int_{a}^{b}[p(s)]_{-} d s}
\end{gathered}
$$

be fulfilled. Then problem (1.3), (1.4) has a unique solution.

Remark 2.1. Let $\lambda \in[1,+\infty[$. Define operator $\psi: L([a, b] ; \mathbb{R}) \rightarrow L([a, b] ; \mathbb{R})$ by the equality

$$
\psi(w)(t) \stackrel{\text { def }}{=} w(a+b-t) \quad \text { for } t \in[a, b] .
$$

Let $\varphi$ be the restriction of $\psi$ to the space $C([a, b] ; \mathbb{R})$. Put

$$
\begin{gathered}
\hat{H}_{0}(w)(t) \stackrel{\text { def }}{=} \psi\left(H_{0}(\varphi(w))\right)(t), \quad \hat{H}_{1}(w)(t) \stackrel{\text { def }}{=} \psi\left(H_{1}(\varphi(w))\right)(t) \quad \text { for } t \in[a, b], \\
\hat{Q}(w)(t) \stackrel{\text { def }}{=}-\psi(Q(\varphi(w)))(t) \quad \text { for } t \in[a, b],
\end{gathered}
$$

and

$$
\hat{h}(w) \stackrel{\text { def }}{=}-\frac{1}{\lambda} h(\varphi(w))
$$


It is clear that if $u$ is a solution of problem (1.1), (1.2), then the function $v \stackrel{\text { def }}{=} \varphi(u)$ is a solution of the problem

$$
v^{\prime}(t)=\hat{H}_{1}(v)(t)-\hat{H}_{0}(v)(t)+\hat{Q}(v)(t), \quad v(a)-\frac{1}{\lambda} v(b)=\hat{h}(v),
$$

and vice versa, if $v$ is a solution of problem (2.29), then the function $u \stackrel{\text { def }}{=} \varphi(v)$ is a solution of problem (1.1), (1.2).

Furthermore, if $H_{i}(i \in\{0,1\})$ is a subadditive (resp., superadditive) operator, then $\hat{H}_{i}(i \in\{0,1\})$ is a subadditive (resp., superadditive) operator, as well.

Theorems 2.5-2.8 and Corollaries 2.3 and 2.4 formulated below can be derived easily from Theorems 2.1-2.4 and Corollaries 2.1 and 2.2, respectively, by using the change of variables described in Remark 2.1.

Theorem 2.5. Let $\lambda \in\left[1,+\infty\left[\right.\right.$ and let $H$ admit the representation $H=H_{0}-H_{1}$, where $H_{0}, H_{1} \in \mathcal{P}_{a b}$. Let, furthermore, (2.1) and (2.2) be fulfilled. If, moreover,

$$
\begin{gathered}
\int_{a}^{b} H_{1}(1)(s) d s<1, \quad \int_{a}^{b}\left|H_{1}(-1)(s)\right| d s<1, \\
\frac{\int_{a}^{b} H_{1}(1)(s) d s}{1-\int_{a}^{b} H_{1}(1)(s) d s}-(\lambda-1)<\int_{a}^{b} H_{0}(1)(s) d s, \\
\frac{\int_{a}^{b}\left|H_{1}(-1)(s)\right| d s}{1-\int_{a}^{b}\left|H_{1}(-1)(s)\right| d s}-(\lambda-1)<\int_{a}^{b}\left|H_{0}(-1)(s)\right| d s,
\end{gathered}
$$

and the inequalities

$$
\begin{aligned}
& {\left[\int_{s}^{t} H_{0}(1)(\xi) d \xi\right.}-1]_{+}\left[\int_{I}\left|H_{0}(-1)(\xi)\right| d \xi-\frac{1}{\lambda}\right]_{+}< \\
&<\left(1-\int_{s}^{t}\left|H_{1}(-1)(\xi)\right| d \xi\right)\left(1-\int_{I} H_{1}(1)(\xi) d \xi\right) \\
& \quad \text { for } s, t \in[a, b], \quad s \leq t,
\end{aligned}
$$

and

$$
\begin{aligned}
{\left[\int_{s}^{t}\left|H_{0}(-1)(\xi)\right| d \xi-1\right]_{+}\left[\int_{I} H_{0}(1)(\xi) d \xi-\frac{1}{\lambda}\right]_{+}<} & \\
<\left(1-\int_{s}^{t} H_{1}(1)(\xi) d \xi\right)\left(1-\int_{I}\left|H_{1}(-1)(\xi)\right| d \xi\right) & \text { for } s, t \in[a, b], \quad s \leq t,
\end{aligned}
$$

are satisfied, where $I=[a, b] \backslash] s, t[$, then problem (1.1), (1.2) has at least one solution. 
Theorem 2.6. Let $\lambda \in\left[1,+\infty\left[\right.\right.$ and let $H$ admit the representation $H=H_{0}-H_{1}$, where $H_{0}, H_{1} \in \mathcal{P}_{a b}$. Let, furthermore, (2.1) and (2.2) be fulfilled. If, moreover,

$$
\begin{gathered}
\int_{a}^{b} H_{0}(1)(s) d s<\frac{1}{\lambda}, \quad \int_{a}^{b}\left|H_{0}(-1)(s)\right| d s<\frac{1}{\lambda}, \\
\frac{\lambda}{1-\lambda \int_{a}^{b} H_{0}(1)(s) d s}-1<\int_{a}^{b} H_{1}(1)(s) d s, \\
\frac{\lambda}{1-\lambda \int_{a}^{b}\left|H_{0}(-1)(s)\right| d s}-1<\int_{a}^{b}\left|H_{1}(-1)(s)\right| d s,
\end{gathered}
$$

and the inequalities

$$
\begin{aligned}
& {\left[\int_{I} H_{1}(1)(\xi) d \xi-1\right]_{+}\left[\int_{s}^{t}\left|H_{1}(-1)(\xi)\right| d \xi-1\right]_{+}<} \\
&<\left(\frac{1}{\lambda}-\int_{I}\left|H_{0}(-1)(\xi)\right| d \xi\right)\left(1-\int_{s}^{t} H_{0}(1)(\xi) d \xi\right) \\
& \quad \text { for } s, t \in[a, b], \quad s \leq t
\end{aligned}
$$

and

$$
\begin{aligned}
& {\left[\int_{I}\left|H_{1}(-1)(\xi)\right| d \xi-1\right]_{+}\left[\int_{s}^{t} H_{1}(1)(\xi) d \xi-1\right]_{+}} \\
& <\left(\frac{1}{\lambda}-\int_{I} H_{0}(1)(\xi) d \xi\right)\left(1-\int_{s}^{t}\left|H_{0}(-1)(\xi)\right| d \xi\right) \\
& \text { for } s, t \in[a, b], \quad s \leq t,
\end{aligned}
$$

are satisfied, where $I=[a, b] \backslash] s, t[$, then problem (1.1), (1.2) has at least one solution.

Theorem 2.7. Let $\lambda \in\left[1,+\infty\left[\right.\right.$ and let $H$ admit the representation $H=H_{0}-H_{1}$, where $H_{0}, H_{1} \in \mathcal{P}_{a b}$. Let, furthermore, each of the operators $H_{0}$ and $H_{1}$ be either a subadditive or a superadditive operator, and let there exist $q \in L([a, b] ; \mathbb{R})$ and $c \in \mathbb{R}$ such that (2.15) and (2.16) are fulfilled. If, moreover,

$$
\begin{gathered}
\int_{a}^{b} \bar{H}_{1}(1)(s) d s<1, \\
\frac{\int_{a}^{b} \bar{H}_{1}(1)(s) d s}{1-\int_{a}^{b} \bar{H}_{1}(1)(s) d s}-(\lambda-1)<\int_{a}^{b}\left|\bar{H}_{0}(-1)(s)\right| d s,
\end{gathered}
$$




$$
\begin{aligned}
& {\left[\int_{s}^{t} \bar{H}_{0}(1)(\xi) d \xi-1\right]_{+}\left[\int_{I} \bar{H}_{0}(1)(\xi) d \xi-\frac{1}{\lambda}\right]_{+} } \\
&<\left(1-\int_{s}^{t} \bar{H}_{1}(1)(\xi) d \xi\right)\left(1-\int_{I} \bar{H}_{1}(1)(\xi) d \xi\right) \\
& \quad \text { for } s, t \in[a, b], \quad s \leq t,
\end{aligned}
$$

where $I=[a, b] \backslash] s, t\left[\right.$, and $\bar{H}_{i}(i=0,1)$ are given by (2.20), then problem (1.1), (1.2) has a unique solution.

Theorem 2.8. Let $\lambda \in\left[1,+\infty\left[\right.\right.$ and let $H$ admit the representation $H=H_{0}-H_{1}$, where $H_{0}, H_{1} \in \mathcal{P}_{a b}$. Let, furthermore, each of the operators $H_{0}$ and $H_{1}$ be either a subadditive or a superadditive operator, and let there exist $q \in L([a, b] ; \mathbb{R})$ and $c \in \mathbb{R}$ such that (2.15) and (2.16) are fulfilled. If, moreover,

$$
\begin{gathered}
\int_{a}^{b} \bar{H}_{0}(1)(s) d s<\frac{1}{\lambda}, \\
\frac{\lambda}{1-\lambda \int_{a}^{b} \bar{H}_{0}(1)(s) d s}-1<\int_{a}^{b}\left|\bar{H}_{1}(-1)(s)\right| d s
\end{gathered}
$$

and

$$
\begin{aligned}
& {\left[\int_{I} \bar{H}_{1}(1)(\xi) d \xi-1\right]_{+}\left[\int_{s}^{t} \bar{H}_{1}(1)(\xi) d \xi-1\right]_{+} } \\
&<\left(\frac{1}{\lambda}-\int_{I} \bar{H}_{0}(1)(\xi) d \xi\right)\left(1-\int_{s}^{t} \bar{H}_{0}(1)(\xi) d \xi\right) \\
& \quad \text { for } s, t \in[a, b], \quad s \leq t,
\end{aligned}
$$

where $I=[a, b] \backslash] s, t\left[\right.$, and $\bar{H}_{i}(i=0,1)$ are given by (2.20), then problem (1.1), (1.2) has a unique solution.

Corollary 2.3. Let $\lambda \in[1,+\infty[$ and let the inequalities

$$
\begin{gathered}
\int_{a}^{b}[p(s)]_{-} d s<1, \\
\frac{\int_{a}^{b}[p(s)]_{-} d s}{1-\int_{a}^{b}[p(s)]_{-} d s}-(\lambda-1)<\int_{a}^{b}[p(s)]_{+} d s<1+\frac{1}{\lambda}+2 \sqrt{1-\int_{a}^{b}[p(s)]_{-} d s}
\end{gathered}
$$

be fulfilled. Then problem (1.3), (1.4) has a unique solution. 
Corollary 2.4. Let $\lambda \in[1,+\infty[$ and let the inequalities

$$
\begin{gathered}
\int_{a}^{b}[p(s)]_{+} d s<\frac{1}{\lambda}, \\
\frac{\lambda}{1-\lambda \int_{a}^{b}[p(s)]_{+} d s}-1<\int_{a}^{b}[p(s)]_{-} d s<2+2 \sqrt{\frac{1}{\lambda}-\int_{a}^{b}[p(s)]_{+} d s}
\end{gathered}
$$

be fulfilled. Then problem (1.3), (1.4) has a unique solution.

\section{Auxiliary propositions}

The following two lemmas are consequences of the result obtained in [31, Corollary 1.4].

Lemma 3.1. Let $\lambda \in[0,1]$ and let the problem

$$
u^{\prime}(t)=\delta H(u)(t), \quad u(a)=\delta \lambda u(b)
$$

have only the trivial solution for every $\delta \in[0,1]$. Let, moreover, (2.1) and (2.2) be fulfilled. Then problem (1.1), (1.2) has at least one solution.

Lemma 3.2. Let $\lambda \in] 0,1]$ and let the problem

$$
u^{\prime}(t)=\delta H(u)(t), \quad \delta u(a)=\lambda u(b)
$$

have only the trivial solution for every $\delta \in[0,1]$. Let, moreover, (2.1) and (2.2) be fulfilled. Then problem (1.1), (1.2) has at least one solution.

Lemma 3.3. Let $\lambda \in] 0,1], F_{i}, G_{i} \in \mathcal{P}_{a b}(i=0,1)$, and let $w \in \tilde{C}([a, b] ; \mathbb{R})$ be $a$ non-trivial function satisfying

$$
\begin{array}{rc}
w^{\prime}(t) \leq \delta F_{0}(w)(t)-\delta F_{1}(w)(t) & \text { for } t \in[a, b], \\
w^{\prime}(t) \geq \delta G_{0}(w)(t)-\delta G_{1}(w)(t) & \text { for } t \in[a, b], \\
w(a)=\delta \lambda w(b) &
\end{array}
$$

for some $\delta \in] 0,1]$. If, moreover,

$$
\begin{gathered}
\int_{a}^{b} F_{0}(1)(s) d s<1, \quad \int_{a}^{b}\left|G_{0}(-1)(s)\right| d s<1, \\
\frac{\int_{a}^{b} F_{0}(1)(s) d s}{1-\int_{a}^{b} F_{0}(1)(s) d s}-\frac{1-\lambda}{\lambda}<\int_{a}^{b} F_{1}(1)(s) d s, \\
\frac{\int_{a}^{b}\left|G_{0}(-1)(s)\right| d s}{1-\int_{a}^{b}\left|G_{0}(-1)(s)\right| d s}-\frac{1-\lambda}{\lambda}<\int_{a}^{b}\left|G_{1}(-1)(s)\right| d s,
\end{gathered}
$$

then there exists $t_{0} \in[a, b]$ such that

$$
w\left(t_{0}\right)=0 .
$$


Proof. Assume that, on the contrary, the function $w$ has no zero. First suppose that

$$
w(t)>0 \quad \text { for } t \in[a, b] .
$$

Put

$$
M=\max \{w(t): t \in[a, b]\}, \quad m=\min \{w(t): t \in[a, b]\}
$$

and choose $t_{M}, t_{m} \in[a, b]$ such that $t_{M} \neq t_{m}$ and

$$
w\left(t_{M}\right)=M, \quad w\left(t_{m}\right)=m .
$$

According to (3.8) and (3.9), we have

$$
M>0, \quad m>0 .
$$

It is obvious that either

$$
t_{M}<t_{m}
$$

or

$$
t_{m}<t_{M}
$$

If (3.12) is fulfilled, then the integration of (3.1) from $a$ to $t_{M}$ and from $t_{m}$ to $b$, respectively, in view of (3.10), yields

$$
\begin{gathered}
M-w(a) \leq \delta \int_{a}^{t_{M}} F_{0}(w)(s) d s-\delta \int_{a}^{t_{M}} F_{1}(w)(s) d s, \\
w(b)-m \leq \delta \int_{t_{m}}^{b} F_{0}(w)(s) d s-\delta \int_{t_{m}}^{b} F_{1}(w)(s) d s .
\end{gathered}
$$

Hence, in view of (3.8), (3.9), and the assumptions $\delta \in] 0,1], \lambda \in] 0,1], F_{0}, F_{1} \in \mathcal{P}_{a b}$, we get

$$
\begin{gathered}
M-w(a) \leq M \int_{a}^{t_{M}} F_{0}(1)(s) d s, \\
\delta \lambda w(b)-m \leq M \int_{t_{m}}^{b} F_{0}(1)(s) d s .
\end{gathered}
$$

Summing the last two inequalities, according to (3.3), (3.4), and (3.11), we obtain

$$
0<M\left(1-\int_{a}^{b} F_{0}(1)(s) d s\right) \leq m .
$$

If (3.13) is fulfilled, then the integration of (3.1) from $t_{m}$ to $t_{M}$, in view of (3.10), yields

$$
M-m \leq \delta \int_{t_{m}}^{t_{M}} F_{0}(w)(s) d s-\delta \int_{t_{m}}^{t_{M}} F_{1}(w)(s) d s .
$$

Hence, on account of (3.8), (3.9), (3.11), and the assumptions $\delta \in] 0,1]$ and $F_{0}, F_{1} \in$ $\mathcal{P}_{a b}$, we get (3.14).

Thus, in both cases (3.12) and (3.13), inequality (3.14) holds. 
On the other hand, the integration of (3.1) from $a$ to $b$ yields

$$
w(b)-w(a) \leq \delta \int_{a}^{b} F_{0}(w)(s) d s-\delta \int_{a}^{b} F_{1}(w)(s) d s,
$$

whence, in view of (3.3), (3.8), (3.9), (3.11), and the assumptions $\delta \in] 0,1], \lambda \in$ ] $0,1]$, and $F_{0}, F_{1} \in \mathcal{P}_{a b}$, we obtain

$$
0 \leq m \delta \frac{1-\lambda}{\lambda} \leq w(b)-w(a) \leq M \delta \int_{a}^{b} F_{0}(1)(s) d s-m \delta \int_{a}^{b} F_{1}(1)(s) d s .
$$

Consequently,

$$
0 \leq m\left(\int_{a}^{b} F_{1}(1)(s) d s+\frac{1-\lambda}{\lambda}\right) \leq M \int_{a}^{b} F_{0}(1)(s) d s .
$$

Multiplying the terms from (3.14) by the respective terms from (3.15), on account of (3.11), we obtain

$$
\int_{a}^{b} F_{1}(1)(s) d s \leq \frac{\int_{a}^{b} F_{0}(1)(s) d s}{1-\int_{a}^{b} F_{0}(1)(s) d s}-\frac{1-\lambda}{\lambda},
$$

which contradicts (3.5).

Now suppose that $w(t)<0$ for $t \in[a, b]$. Then, according to (3.2) and (3.3), $v \stackrel{\text { def }}{=}-w$ is a non-trivial function satisfying

$$
v^{\prime}(t) \leq \delta \tilde{F}_{0}(v)(t)-\delta \tilde{F}_{1}(v)(t) \quad \text { for } t \in[a, b], \quad v(a)=\delta \lambda v(b),
$$

where

$$
\tilde{F}_{i}(v)(t)=-G_{i}(-v)(t) \quad \text { for } t \in[a, b] \quad(i=0,1) .
$$

Obviously, $\tilde{F}_{0}, \tilde{F}_{1} \in \mathcal{P}_{a b}$, and $v(t)>0$ for $t \in[a, b]$. Therefore, following the steps taken above we get

$$
\int_{a}^{b}\left|G_{1}(-1)(s)\right| d s \leq \frac{\int_{a}^{b}\left|G_{0}(-1)(s)\right| d s}{1-\int_{a}^{b}\left|G_{0}(-1)(s)\right| d s}-\frac{1-\lambda}{\lambda},
$$

which contradicts (3.6).

Lemma 3.4. Let $\lambda \in[0,1], F_{i}, G_{i} \in \mathcal{P}_{a b}(i=0,1)$, and let $w \in \tilde{C}([a, b] ; \mathbb{R})$ be a nontrivial function satisfying (3.1)-(3.3) for some $\delta \in] 0,1]$. If, moreover, inequalities (3.4),

$$
\begin{aligned}
{\left[\int_{s}^{t} G_{1}(1)(\xi) d \xi-1\right]_{+}\left[\int_{I}\left|F_{1}(-1)(\xi)\right| d \xi-\lambda\right]_{+}<} & \\
<\left(1-\int_{s}^{t}\left|G_{0}(-1)(\xi)\right| d \xi\right)\left(1-\int_{I} F_{0}(1)(\xi) d \xi\right) & \text { for } s, t \in[a, b], \quad s \leq t,
\end{aligned}
$$


and

$$
\begin{aligned}
{\left[\int_{s}^{t}\left|F_{1}(-1)(\xi)\right| d \xi-1\right]_{+}\left[\int_{I} G_{1}(1)(\xi) d \xi-\lambda\right]_{+}<} \\
<\left(1-\int_{s}^{t} F_{0}(1)(\xi) d \xi\right)\left(1-\int_{I}\left|G_{0}(-1)(\xi)\right| d \xi\right) \\
\quad \text { for } s, t \in[a, b], \quad s \leq t
\end{aligned}
$$

are fulfilled, where $I=[a, b] \backslash] s, t[$, then

$$
w(t) \neq 0 \quad \text { for } t \in[a, b] .
$$

Proof. Assume that, on the contrary, there exists a point $t_{0} \in[a, b]$ such that (3.7) is fulfilled. Put

$$
M=\max \{w(t): t \in[a, b]\}, \quad m=-\min \{w(t): t \in[a, b]\}
$$

and choose $t_{M}, t_{m} \in[a, b]$ such that $t_{M} \neq t_{m}$ and

$$
w\left(t_{M}\right)=M, \quad w\left(t_{m}\right)=-m
$$

According to (3.7) and (3.19) we have

$$
M \geq 0, \quad m \geq 0, \quad M+m>0 .
$$

Obviously, either (3.12) or (3.13) is fulfilled.

Suppose that (3.12) is satisfied. Then the integration of (3.1) from $a$ to $t_{M}$ and from $t_{m}$ to $b$, and the integration of (3.2) from $t_{M}$ to $t_{m}$, respectively, by virtue of (3.20), yields

$$
\begin{gathered}
M-w(a) \leq \delta \int_{a}^{t_{M}} F_{0}(w)(s) d s-\delta \int_{a}^{t_{M}} F_{1}(w)(s) d s \\
-m-M \geq \delta \int_{t_{M}}^{t_{m}} G_{0}(w)(s) d s-\delta \int_{t_{M}}^{t_{m}} F_{1}(w)(s) d s \\
w(b)+m \leq \delta \int_{t_{m}}^{b} F_{0}(w)(s) d s-\delta \int_{t_{m}}^{b} F_{1}(w)(s) d s .
\end{gathered}
$$

Hence, in view of (3.19), (3.21), and the assumptions $\delta \in] 0,1], \lambda \in[0,1], F_{i}, G_{i} \in$ $\mathcal{P}_{a b}(i=0,1)$, we get

$$
\begin{gathered}
\delta M-w(a) \leq \delta M \int_{a}^{t_{M}} F_{0}(1)(s) d s+\delta m \int_{a}^{t_{M}}\left|F_{1}(-1)(s)\right| d s \\
M+m \leq m \int_{t_{M}}^{t_{m}}\left|G_{0}(-1)(s)\right| d s+M \int_{t_{M}}^{t_{m}} G_{1}(1)(s) d s \\
\delta \lambda(w(b)+m) \leq \delta M \int_{t_{m}}^{b} F_{0}(1)(s) d s+\delta m \int_{t_{m}}^{b}\left|F_{1}(-1)(s)\right| d s .
\end{gathered}
$$


Summing (3.22) and (3.24), in view of (3.3), (3.4), (3.21), and the assumption $\delta>0$, we obtain

$$
0 \leq M\left(1-\int_{I} F_{0}(1)(s) d s\right) \leq m\left[\int_{I}\left|F_{1}(-1)(s)\right| d s-\lambda\right]_{+},
$$

where $I=[a, b] \backslash] t_{M}, t_{m}[$. Further on, (3.23) according to (3.4) and (3.21), results in

$$
0 \leq m\left(1-\int_{t_{M}}^{t_{m}}\left|G_{0}(-1)(s)\right| d s\right) \leq M\left[\int_{t_{M}}^{t_{m}} G_{1}(1)(s) d s-1\right]_{+} .
$$

Now from (3.25) and (3.26) it follows that $M>0, m>0$. Therefore, multiplying (3.25) and (3.26) we obtain

$$
\begin{aligned}
\left(1-\int_{I} F_{0}(1)(s) d s\right)\left(1-\int_{t_{M}}^{t_{m}}\right. & \left.\left|G_{0}(-1)(s)\right| d s\right) \leq \\
\leq & \leq\left[\int_{I}\left|F_{1}(-1)(s)\right| d s-\lambda\right]_{+}\left[\int_{t_{M}}^{t_{m}} G_{1}(1)(s) d s-1\right]_{+},
\end{aligned}
$$

which contradicts (3.16).

Now suppose that (3.13) holds. Then, according to (3.1)-(3.3), $v \stackrel{\text { def }}{=}-w$ is a nontrivial function satisfying the relations

$$
\begin{array}{cc}
v^{\prime}(t) \leq \delta \tilde{F}_{0}(v)(t)-\delta \tilde{F}_{1}(v)(t) & \text { for } t \in[a, b], \\
v^{\prime}(t) \geq \delta \tilde{G}_{0}(v)(t)-\delta \tilde{G}_{1}(v)(t) & \text { for } t \in[a, b], \\
v(a)=\delta \lambda v(b), &
\end{array}
$$

where

$$
\tilde{F}_{i}(v)(t)=-G_{i}(-v)(t), \quad \tilde{G}_{i}(v)(t)=-F_{i}(-v)(t) \quad \text { for } t \in[a, b] \quad(i=0,1) .
$$

Obviously, $\tilde{F}_{i}, \tilde{G}_{i} \in \mathcal{P}_{a b}(i=0,1)$, and

$$
v\left(t_{m}\right)=\max \{v(t): t \in[a, b]\}, \quad v\left(t_{M}\right)=\min \{v(t): t \in[a, b]\} .
$$

Therefore, the substitution $v \stackrel{\text { def }}{=}-w$ transforms the case considered into the previous one and, following the steps above, we get

$$
\begin{aligned}
\left(1-\int_{I}\left|G_{0}(-1)(s)\right| d s\right)\left(1-\int_{t_{m}}^{t_{M}} F_{0}(1)(s) d s\right) \leq \\
\quad \leq\left[\int_{I} G_{1}(1)(s) d s-\lambda\right]_{+}\left[\int_{t_{m}}^{t_{M}}\left|F_{1}(-1)(s)\right| d s-1\right]_{+},
\end{aligned}
$$

where $I=[a, b] \backslash] t_{m}, t_{M}[$, which contradicts (3.17).

Lemma 3.5. Let $\lambda \in] 0,1], F_{i}, G_{i} \in \mathcal{P}_{a b}(i=0,1)$, and let $w \in \tilde{C}([a, b] ; \mathbb{R})$ be $a$ non-trivial function satisfying inequalities (3.1), (3.2), and

$$
\delta w(a)=\lambda w(b)
$$


for some $\delta \in] 0,1]$. If, moreover,

$$
\begin{gathered}
\int_{a}^{b} G_{1}(1)(s) d s<\lambda, \quad \int_{a}^{b}\left|F_{1}(-1)(s)\right| d s<\lambda, \\
\frac{1}{\lambda-\int_{a}^{b} G_{1}(1)(s) d s}-1<\int_{a}^{b} G_{0}(1)(s) d s, \\
\frac{1}{\lambda-\int_{a}^{b}\left|F_{1}(-1)(s)\right| d s}-1<\int_{a}^{b}\left|F_{0}(-1)(s)\right| d s,
\end{gathered}
$$

then there exists a point $t_{0} \in[a, b]$ such that (3.7) holds.

Proof. Assume that, on the contrary, the function $w$ has no zero. First suppose that (3.8) is fulfilled. Define numbers $M$ and $m$ by (3.9) and choose $t_{M}, t_{m} \in[a, b]$ such that $t_{M} \neq t_{m}$ and (3.10) is satisfied. According to (3.8) and (3.9) we have (3.11). Obviously, either (3.12) or (3.13) holds.

If (3.13) is fulfilled, then the integration of (3.2) from $a$ to $t_{m}$ and from $t_{M}$ to $b$, respectively, in view of (3.10), yields

$$
\begin{gathered}
w(a)-m \leq-\delta \int_{a}^{t_{m}} G_{0}(w)(s) d s+\delta \int_{a}^{t_{m}} G_{1}(w)(s) d s, \\
M-w(b) \leq-\delta \int_{t_{M}}^{b} G_{0}(w)(s) d s+\delta \int_{t_{M}}^{b} G_{1}(w)(s) d s .
\end{gathered}
$$

Hence, in view of (3.8), (3.9), and the assumptions $\delta \in] 0,1], \lambda \in] 0,1], G_{0}, G_{1} \in$ $\mathcal{P}_{a b}$, we get

$$
\begin{aligned}
& \delta(w(a)-m) \leq M \int_{a}^{t_{m}} G_{1}(1)(s) d s, \\
& \lambda(M-w(b)) \leq M \int_{t_{M}}^{b} G_{1}(1)(s) d s .
\end{aligned}
$$

Summing the last two inequalities, and taking (3.11), (3.27), and (3.28) into account, we obtain

$$
0<M\left(\lambda-\int_{a}^{b} G_{1}(1)(s) d s\right) \leq \delta m .
$$

If (3.12) is fulfilled, then the integration of (3.2) from $t_{M}$ to $t_{m}$, in view of (3.10), yields

$$
M-m \leq-\delta \int_{t_{M}}^{t_{m}} G_{0}(w)(s) d s+\delta \int_{t_{M}}^{t_{m}} G_{1}(w)(s) d s .
$$

Suppose that $\lambda \leq \delta$. Then from (3.32), in view of (3.8), (3.9), and the assumptions $\delta \in] 0,1], \lambda \in] 0,1]$, and $G_{0}, G_{1} \in \mathcal{P}_{a b}$, it follows that

$$
\lambda M-\delta m \leq \lambda(M-m) \leq M \int_{a}^{b} G_{1}(1)(s) d s,
$$


whence we get (3.31). If $\delta<\lambda$, then from (3.32), in view of (3.8), (3.9), and the assumptions $\delta \in] 0,1], \lambda \in] 0,1]$, and $G_{0}, G_{1} \in \mathcal{P}_{a b}$, we obtain

$$
0<M\left(\lambda-\int_{a}^{b} G_{1}(1)(s) d s\right) \leq m .
$$

Thus in both cases (3.12) and (3.13), we have

$$
\begin{array}{ll}
0<M\left(\lambda-\int_{a}^{b} G_{1}(1)(s) d s\right) \leq \delta m & \text { if } \lambda \leq \delta, \\
0<M\left(\lambda-\int_{a}^{b} G_{1}(1)(s) d s\right) \leq m & \text { if } \lambda>\delta .
\end{array}
$$

On the other hand, the integration of (3.2) from $a$ to $b$ yields

$$
w(a)-w(b) \leq-\delta \int_{a}^{b} G_{0}(w)(s) d s+\delta \int_{a}^{b} G_{1}(w)(s) d s .
$$

If now $\lambda \leq \delta$, then from (3.35), on account of (3.8), (3.9), (3.11), (3.27), and the assumptions $\delta \in] 0,1], \lambda \in] 0,1]$, and $G_{0}, G_{1} \in \mathcal{P}_{a b}$, we obtain

$$
-M(1-\lambda) \leq w(b)(\lambda-1) \leq w(a)-w(b) \leq-m \delta \int_{a}^{b} G_{0}(1)(s) d s+M \int_{a}^{b} G_{1}(1)(s) d s .
$$

Therefore,

$$
0 \leq m \delta \int_{a}^{b} G_{0}(1)(s) d s \leq M\left(\int_{a}^{b} G_{1}(1)(s) d s+1-\lambda\right) \quad \text { if } \lambda \leq \delta .
$$

If $\lambda>\delta$, from (3.35), on account of (3.8), (3.11), (3.27), and the assumption $\lambda \in$ ]0, 1], we have

$$
-M \delta(1-\lambda) \leq 0 \leq w(a)-w(b) \leq-m \delta \int_{a}^{b} G_{0}(1)(s) d s+M \delta \int_{a}^{b} G_{1}(1)(s) d s .
$$

Hence, in view of the assumption $\delta>0$, we get

$$
0 \leq m \int_{a}^{b} G_{0}(1)(s) d s \leq M\left(\int_{a}^{b} G_{1}(1)(s) d s+1-\lambda\right) \quad \text { if } \lambda>\delta .
$$

Multiplying the terms in (3.33) (resp., (3.34)) by the corresponding terms in (3.36) (resp., (3.37)), on account of (3.11) and the assumption $\delta>0$, we obtain

$$
\int_{a}^{b} G_{0}(1)(s) d s \leq \frac{1}{\lambda-\int_{a}^{b} G_{1}(1)(s) d s}-1,
$$

which contradicts (3.29).

Now suppose that $w(t)<0$ for $t \in[a, b]$. Then, according to (3.1) and (3.27), $v \stackrel{\text { def }}{=}-w$ is a non-trivial function satisfying

$$
v^{\prime}(t) \geq \delta \tilde{G}_{0}(v)(t)-\delta \tilde{G}_{1}(v)(t) \quad \text { for } t \in[a, b], \quad \delta v(a)=\lambda v(b),
$$


where

$$
\tilde{G}_{i}(v)(t)=-F_{i}(-v)(t) \quad \text { for } t \in[a, b] \quad(i=0,1) .
$$

Obviously, $\tilde{G}_{0}, \tilde{G}_{1} \in \mathcal{P}_{a b}$, and $v(t)>0$ for $t \in[a, b]$. Therefore, following the steps taken above we get

$$
\int_{a}^{b}\left|F_{0}(-1)(s)\right| d s \leq \frac{1}{\lambda-\int_{a}^{b}\left|F_{1}(-1)(s)\right| d s}-1,
$$

which contradicts (3.30).

Lemma 3.6. Let $\lambda \in] 0,1], F_{i}, G_{i} \in \mathcal{P}_{a b}(i=0,1)$, and let $w \in \tilde{C}([a, b] ; \mathbb{R})$ be a nontrivial function satisfying (3.1), (3.2), and (3.27) for some $\delta \in] 0,1]$. If, moreover, inequalities (3.28),

$$
\begin{aligned}
{\left[\int_{I} F_{0}(1)(\xi) d \xi-1\right]_{+}\left[\int_{s}^{t}\left|G_{0}(-1)(\xi)\right| d \xi-1\right]_{+}<} \\
<\left(\lambda-\int_{I}\left|F_{1}(-1)(\xi)\right| d \xi\right)\left(1-\int_{s}^{t} G_{1}(1)(\xi) d \xi\right) \\
\quad \text { for } s, t \in[a, b], \quad s \leq t
\end{aligned}
$$

and

$$
\begin{aligned}
& {\left[\int_{I}\left|G_{0}(-1)(\xi)\right| d \xi\right.}-1]_{+}\left[\int_{s}^{t} F_{0}(1)(\xi) d \xi-1\right]_{+}< \\
&<\left(\lambda-\int_{I} G_{1}(1)(\xi) d \xi\right)\left(1-\int_{s}^{t}\left|F_{1}(-1)(\xi)\right| d \xi\right) \\
& \text { for } s, t \in[a, b], \quad s \leq t,
\end{aligned}
$$

are fulfilled, where $I=[a, b] \backslash] s, t[$, then (3.18) holds.

Proof. Assume that, on the contrary, there exists $t_{0} \in[a, b]$ such that (3.7) holds. Define numbers $M$ and $m$ by (3.19) and choose $t_{M}, t_{m} \in[a, b]$ such that $t_{M} \neq t_{m}$ and (3.20) is fulfilled. According to (3.7) and (3.19) we have (3.21). Obviously, either (3.12) or (3.13) is fulfilled.

Suppose that (3.12) is satisfied. Then the integration of (3.1) from $a$ to $t_{M}$ and from $t_{m}$ to $b$, and the integration of (3.2) from $t_{M}$ to $t_{m}$, respectively, by virtue of (3.20), yields

$$
\begin{gathered}
M-w(a) \leq \delta \int_{a}^{t_{M}} F_{0}(w)(s) d s-\delta \int_{a}^{t_{M}} F_{1}(w)(s) d s, \\
-m-M \geq \delta \int_{t_{M}}^{t_{m}} G_{0}(w)(s) d s-\delta \int_{t_{M}}^{t_{m}} G_{1}(w)(s) d s, \\
w(b)+m \leq \delta \int_{t_{m}}^{b} F_{0}(w)(s) d s-\delta \int_{t_{m}}^{b} F_{1}(w)(s) d s .
\end{gathered}
$$


Hence, in view of (3.19), (3.21), and the assumptions $\delta \in] 0,1], \lambda \in] 0,1], F_{i}, G_{i} \in$ $\mathcal{P}_{a b}(i=0,1)$, we get

$$
\begin{gathered}
\delta(M-w(a)) \leq \delta M \int_{a}^{t_{M}} F_{0}(1)(s) d s+\delta m \int_{a}^{t_{M}}\left|F_{1}(-1)(s)\right| d s, \\
M+m \leq m \int_{t_{M}}^{t_{m}}\left|G_{0}(-1)(s)\right| d s+M \int_{t_{M}}^{t_{m}} G_{1}(1)(s) d s, \\
\lambda(w(b)+\delta m) \leq \delta M \int_{t_{m}}^{b} F_{0}(1)(s) d s+\delta m \int_{t_{m}}^{b}\left|F_{1}(-1)(s)\right| d s .
\end{gathered}
$$

Summing (3.40) and (3.42), according to (3.21), (3.27), (3.28), and the assumption $\delta>0$, we obtain

$$
0 \leq m\left(\lambda-\int_{I}\left|F_{1}(-1)(s)\right| d s\right) \leq M\left[\int_{I} F_{0}(1)(s) \mid d s-1\right]_{+},
$$

where $I=[a, b] \backslash] t_{M}, t_{m}[$. Furthermore, (3.41) in view of (3.28) and (3.21), results in

$$
0 \leq M\left(1-\int_{t_{M}}^{t_{m}} G_{1}(1)(s) d s\right) \leq m\left[\int_{t_{M}}^{t_{m}}\left|G_{0}(-1)(s)\right| d s-1\right]_{+} .
$$

Now from (3.43) and (3.44) it follows that $M>0, m>0$. Therefore, multiplying the terms in (3.43) by the corresponding terms in (3.44), we obtain

$$
\begin{aligned}
\left(\lambda-\int_{I}\left|F_{1}(-1)(s)\right| d s\right)\left(1-\int_{t_{M}}^{t_{m}} G_{1}(1)(s) d s\right) \leq \\
\quad \leq\left[\int_{I} F_{0}(1)(s) d s-1\right]_{+}\left[\int_{t_{M}}^{t_{m}}\left|G_{0}(-1)(s)\right| d s-1\right]_{+},
\end{aligned}
$$

which contradicts (3.38).

Now suppose that (3.13) holds. Then, according to (3.1), (3.2), and (3.27), $v \stackrel{\text { def }}{=}-w$ is a non-trivial function satisfying

$$
\begin{array}{cc}
v^{\prime}(t) \leq \delta \tilde{F}_{0}(v)(t)-\delta \tilde{F}_{1}(v)(t) & \text { for } t \in[a, b], \\
v^{\prime}(t) \geq \delta \tilde{G}_{0}(v)(t)-\delta \tilde{G}_{1}(v)(t) & \text { for } t \in[a, b], \\
\delta v(a)=\lambda v(b), &
\end{array}
$$

where

$$
\tilde{F}_{i}(v)(t)=-G_{i}(-v)(t), \quad \tilde{G}_{i}(v)(t)=-F_{i}(-v)(t) \quad \text { for } t \in[a, b] \quad(i=0,1) .
$$

Obviously, $\tilde{F}_{i}, \tilde{G}_{i} \in \mathcal{P}_{a b}(i=0,1)$, and

$$
v\left(t_{m}\right)=\max \{v(t): t \in[a, b]\}, \quad v\left(t_{M}\right)=\min \{v(t): t \in[a, b]\} .
$$


Therefore, the substitution $v \stackrel{\text { def }}{=}-w$ transforms the case considered into the previous one and, arguing similarly to the considerations above, we get

$$
\begin{aligned}
\left(\lambda-\int_{I} G_{1}(1)(s) d s\right)\left(1-\int_{t_{m}}^{t_{M}}\right. & \left.\left|F_{1}(-1)(s)\right| d s\right) \leq \\
\leq & {\left[\int_{I}\left|G_{0}(-1)(s)\right| d s-1\right]_{+}\left[\int_{t_{m}}^{t_{M}} F_{0}(1)(s) d s-1\right]_{+}, }
\end{aligned}
$$

where $I=[a, b] \backslash] t_{m}, t_{M}[$, which contradicts (3.39).

\section{Proofs}

Theorem 2.1 follows from Lemmas 3.1, 3.3, and 3.4, Theorem 2.1' follows from Lemmas 3.1 and 3.4, and Theorem 2.2 follows from Lemmas 3.2, 3.5, and 3.6.

Proof of Theorem 2.3. Let us first note that, for every operator $T \in \mathcal{H}_{a b}$ and arbitrary $t \in[a, b]$ and $v \in C([a, b] ; \mathbb{R})$, we have

$$
-T(-v)(t) \leq T(v)(t)
$$

if $T$ is subadditive, and

$$
T(v)(t) \leq-T(-v)(t)
$$

if $T$ is superadditive. Therefore,

$$
-\bar{H}_{i}(-1)(t) \leq \bar{H}_{i}(1)(t) \quad \text { for } t \in[a, b] \quad(i=0,1),
$$

and, consequently, inequalities (2.17)-(2.19) yield inequalities (2.3)-(2.7). Thus, the assumptions of Theorem 2.1 are fulfilled, and problem (1.1), (1.2) has at least one solution.

We will show that (1.1), (1.2) has no more than one solution. Let $u$ and $v$ be solutions of (1.1), (1.2). Then, according to (2.15) and (2.16), the function $w(t)=$ $u(t)-v(t)$ for $t \in[a, b]$ satisfies (3.1)-(3.3) with $\delta=1$ and

$$
\begin{array}{rrr}
F_{0}(w)(t)=\bar{H}_{0}(w)(t), & F_{1}(w)(t)=-\bar{H}_{1}(-w)(t) & \text { for } t \in[a, b], \\
G_{0}(w)(t)=-\bar{H}_{0}(-w)(t), & G_{1}(w)(t)=\bar{H}_{1}(w)(t) & \text { for } t \in[a, b] .
\end{array}
$$

According to Lemmas 3.3 and 3.4 we have $w \equiv 0$, i. e., $u \equiv v$.

Theorems $2.3^{\prime}$ and 2.4 can be proved analogously.

Proof of Corollary 2.1. Let us put

$$
\begin{array}{cc}
H_{0}(v)(t) \stackrel{\text { def }}{=}[p(t)]_{+} \max \left\{v(s): \tau_{1}(t) \leq s \leq \tau_{2}(t)\right\} & \text { for } t \in[a, b], \\
H_{1}(v)(t) \stackrel{\text { def }}{=}[p(t)]_{-} \max \left\{v(s): \tau_{1}(t) \leq s \leq \tau_{2}(t)\right\} & \text { for } t \in[a, b], \\
Q(v)(t) \stackrel{\text { def }}{=} q(t) \quad \text { for } t \in[a, b], &
\end{array}
$$


and $h(v) \stackrel{\text { def }}{=} c$. Then the assumptions of Theorem 2.3 are fulfilled because

$$
\begin{aligned}
{\left[\int_{s}^{t} \bar{H}_{1}(1)(\xi) d \xi-1\right]_{+} } & {\left[\int_{I} \bar{H}_{1}(1)(\xi) d \xi-\lambda\right]_{+} \leq } \\
\leq & \frac{1}{4}\left[\int_{a}^{b}[p(s)]_{-} d s-1-\lambda\right]_{+}^{2} \quad \text { for } s, t \in[a, b], \quad s \leq t
\end{aligned}
$$

and

$$
\begin{aligned}
\left(1-\int_{s}^{t} \bar{H}_{0}(1)(\xi) d \xi\right)\left(1-\int_{I} \bar{H}_{0}(1)(\xi) d \xi\right) \geq & \\
& \geq 1-\int_{a}^{b}[p(s)]_{+} d s \quad \text { for } s, t \in[a, b], \quad s \leq t,
\end{aligned}
$$

where $I=[a, b] \backslash] s, t[$.

In a similar way, it can be proved that Corollary $2.1^{\prime}$ follows from Theorem $2.3^{\prime}$ and Corollary 2.2 follows from Theorem 2.4.

\section{ACKNOWLedgement}

This work was supported by the Grant No. 201/00/D058 of the Grant Agency of the Czech Republic.

\section{REFERENCES}

[1] Azbelev, N. V., Maksimov, V. P., and Rakhmatullina, L. F.: Introduction to the Theory of Functional Differential Equations [in Russian], Nauka, Moscow, 1991.

[2] Azbelev, N. V. and Rakhmatullina, L. F.: Theory of linear abstract functional differential equations and applications, Mem. Differential Equations Math. Phys., 8 (1996), 1-102.

[3] Bantsur, N. R. AND Trofimchuк, E. P.: On existence and stability of periodic and almost-periodic solutions of quasilinear equations with maxima, Nonlinear Oscillations, 1 (1998), No. 1, 1-5.

[4] Bantsur, N. R. and Trofimchuк, E. P.: On the existence of T-periodic solutions to essentially nonlinear scalar differential equations with maxima, Ukrain. Math. J., 50 (1998), No. 6, 747-754.

[5] BRavyi, E., HaKL, R., and Lomtatidze, A.: On Cauchy problem for the first order nonlinear functional differential equations of non-Volterra's type, Czechoslovak Math. J., 52 (2002), No. 4, 673-690.

[6] Bravyi, E., Hakl, R., and Lomtatidze, A.: Optimal conditions for unique solvability of the Cauchy problem for first order linear functional differential equations, Czechoslovak Math. J., 52 (2002), No. 3, 513-530.

[7] Gelashvili, S. M.: On a boundary value problem for systems of functional differential equations, Arch. Math. (Brno), 20 (1984), 157-168.

[8] HAKL, R.: On some boundary value problems for systems of linear functional differential equations, El. J. Qualitative Theory of Diff. Equations (1999), No. 10, 1-16.

[9] HaKL, R., Kiguradze I., ANd PŮžA, B.: Upper and lower solutions of boundary value problems for functional differential equations and theorems on functional differential inequalities, Georgian Math. J., 7 (2000), No. 3, 489-512. 
[10] Hakl, R. and Lomtatidze, A.: A note on the Cauchy problem for first order linear differential equations with a deviating argument, Arch. Math. (Brno), 38 (2002), No. 1, 61-71.

[11] Hakl, R., Lomtatidze, A., And PŮžA, B.: New optimal conditions for unique solvability of the Cauchy problem for first order linear functional differential equations, Math. Bohemica, 127 (2002), No. 4, 509-524.

[12] Hakl, R., Lomtatidze, A., AND PŮŽA, B.: On a boundary value problem for first order scalar functional differential equations, Nonlin. Anal.: Theory, Meth. \& Appl., 53 (2003), Nos. 3-4, 391-405.

[13] HAKL, R., Lomtatidze, A., AND PŮŽA, B.: On nonnegative solutions of first order scalar functional differential equations, Mem. Differential Equations Math. Phys., 23 (2001), 51-84.

[14] Hakl, R., Lomtatidze, A., AND PU゚ŽA, B.: On periodic solutions of first order linear functional differential equations, Nonlinear Anal.: Theory, Meth. \& Appl., 49 (2002), No. 7, 929-945.

[15] Hakl, R., Lomtatidze, A., AND PŮŽA, B.: On periodic solutions of first order nonlinear functional differential equations of non-Volterra's type, Mem. Differential Equations Math. Phys., 24 (2001), 83-105.

[16] HaKL, R., Lomtatidze, A., And ŠRemR, J.: On an antiperiodic type boundary value problem for first order linear functional differential equations, Arch. Math. (Brno), 38 (2002), No. 2, 149-160.

[17] HaKL, R., Lomtatidze, A., AND ŠRemR, J.: On a periodic type boundary value problem for first order linear functional differential equations, Nonlin. Oscillations, 5 (2002), No. 3, 416-432.

[18] HaKL, R., LomTatidze, A., AND ŠREMR, J.: On a periodic-type boundary value problem for firstorder nonlinear functional differential equations, Nonlin. Anal.: Theory, Meth. \& Appl., 51 (2002), No. 3, 425-447.

[19] HAKL, R., LomtatidZe, A., AND ŠRemR, J.: On constant sign solutions of a periodic type boundary value problems for first order scalar functional differential equations, Mem. Differential Equations Math. and Phys., 26 (2002), 65-90.

[20] HaKL, R., Lomtatidze, A., AND Šremr, J.: Solvability and unique solvability of a periodic type boundary value problem for first order functional differential equations, Georgian Math. J., 9 (2002), No. 3, 525-547.

[21] HAKL, R., LomTATIDZE, A., AND ŠREMR, J.: Solvability of a periodic type boundary value problem for first order functional differential equations, Arch. Math. (Brno), to appear.

[22] Hakl, R., Lomtatidze, A., and Šremr, J.: Some Boundary Value Problems for First Order Scalar Functional Differential Equations. Folia Facult. Scien. Natur. Univ. Masar. Brunensis, Brno, 2002.

[23] Hale, J.: Theory of Functional Differential Equations, Springer-Verlag, New York-HeidelbergBerlin, 1977.

[24] Kiguradze I. and PŮžA, B.: Boundary Value Problems for Systems of Linear Functional Differential Equations, Folia Facult. Scien. Natur. Univ. Masar. Brunensis, Brno, 2003.

[25] Kiguradze I. and PŮža, B.: Conti-Opial type theorems for systems of functional differential equations, Differents. Uravn., 33 (1997), No. 2, 185-194.

[26] Kiguradze I. And PŮžA, B.: On boundary value problems for functional differential equations, Mem. Differential Equations Math. Phys., 12 (1997), 106-113.

[27] Kiguradze I. AND PŮžA, B.: On boundary value problems for systems of linear functional differential equations, Czechoslovak Math. J., 47 (1997), No. 2, 341-373.

[28] Kiguradze I. and PŮža, B.: On periodic solutions of systems of linear functional differential equations, Arch. Math. (Brno), 33 (1997), No. 3, 197-212.

[29] Kiguradze I. AND PŮžA, B.: On the solvability of boundary value problems for systems of nonlinear differential equations with deviating arguments, Mem. Differential Equations Math. Phys., 10 (1997), 157-161.

[30] Kiguradze I. AND PŮžA, B.: On the solvability of nonlinear boundary value problems for functional differential equations, Georgian Math. J., 5 (1998), No. 3, 251-262. 
[31] Kiguradze I., PŮža, B., and Stavroulakis, I. P.: On singular boundary value problems for functional differential equations of higher order, Georgian Math. J., 8 (2001), No. 4, 791-814.

[32] KolmanovskiI, V. And Myshisis, A.: Introduction to the Theory and Applications of Functional Differential Equations, Kluwer Academic Publishers, 1999.

[33] Liz, E. AND TrofimchuK, S.: Existence and stability of almost periodic solutions for quasilinear delay systems and Halanay inequality, J. Math. Anal. Appl., 248 (2000), 625-644.

[34] Lomtatidze, A., PŮžA, B., AND HaKL, R.: On a periodic boundary value problem for the first order scalar functional differential equation, Differents. Uravn., 39 (2003), No. 3, 320-327.

[35] Magomedov, A. R.: Theorems on existence and uniqueness of solutions of differential equations with maximal functional parameters, Arch. Math. (Brno), 28 (1992), 139-154.

[36] Pinto, M. AND Trofimchuк, S.: Stability and existence of multiple periodic solutions for a quasilinear differential equation with maxima, Proc. Roy. Soc. Edinburgh Sect. A, 130 (2000), 1103-1118.

[37] PŮžA, B.: Some boundary value problems for nonlinear functional-differential equations, Differents. Uravn., 37 (2001), No. 6, 761-770.

[38] Ronto, A. N.: On the periodic solutions of systems with "maxima”: Dopov. Nats. Akad. Nauk Ukr., 1999, No. 12, 27-30.

[39] Ronto, A. And Samoilenko, A.: On the unique solvability of some linear boundary value problems. In the book "Nonlinear Analysis and Applications" (R. P. Agarwal and D. O’Regan, eds.), Vol. II, Kluwer Academic Publishers, Dordrecht, 2003.

[40] Schwabik, Š, TvrdÝ, M., and Vejvoda, O.: Differential and Integral Equations: Boundary Value Problems and Adjoints, Academia, Praha, 1979.

[41] Stepanov E.: On solvability of some boundary value problems for differential equations with "maxima”, Topol. Methods Nonlinear Anal., 8 (1996), 315-326.

[42] Tsitskishvili R. A.: Unique solvability and correctness of a linear boundary value problem for functional-differential equations, Rep. Enlarged Sessions Sem. I. N. Vekua Inst. Appl. Math., 5 (1990), No. 3, 195-198.

\section{Author's Address}

\section{Robert Hakl:}

Mathematical Institute, Academy of Sciences of the Czech Republic, ŽižKova 22, 61662 Brno, Czech Republic

E-mail address: hakl@ipm.cz 